\title{
Effect of Segmented Sleeping on the Academic Performance of Medical Students. A Questionnaire Survey
}

\author{
Shalini S.P. ${ }^{1}$, Balaji K. ${ }^{2}$, Yuvaraj Maria Francis ${ }^{3}$, Gunapriya Raghunath ${ }^{4}$, Vijayalakshmi S. ${ }^{5}$ \\ ${ }^{1}$ Phase I MBBS, Saveetha Medical College and Hospital, Thandalam, Chennai, India, ${ }^{2}$ Tutor, Department of \\ Anatomy, Saveetha Medical College and Hospital, Thandalam, Chennai, India, ${ }^{3}$ Assistant Professor, Department \\ of Anatomy, Saveetha Medical College and Hospital, Thandalam, Chennai, India, ${ }^{4}$ Professor \& Head, Department \\ of Anatomy, Saveetha Medical College and Hospital, Thandalam, Chennai, India, ${ }^{5}$ Professor, Department of \\ Anatomy, Saveetha Medical College and Hospital, Thandalam, Chennai, India
}

\begin{abstract}
Background: Sleep is a basic need of every individual, essential for a better quality of life and performance in the activity of daily living. Humans spend a third of their life sleeping. Even then, sleep disturbance is the common health issue in modern society which promotes late-night gatherings and shift-based work. Segmented sleep is one or many naps with one core sleep where the awake period also takes place in two or more sessions. Segmented sleep is considered to be an unhealthy practice today, is the way that our ancestors slept until the last few centuries.
\end{abstract}

Aim and Objectives: The study aims to assess the effect of segmented sleep patterns among medical students and correlating it with the academic performance of them.

Method and Materials: This study was done on 300 students with both male and female participants. A questionnaire was developed after the elaborated discussion and circulated among the students. The data was then analyzed using Microsoft Excel 2010.

Results: The overall quality of the sleep, regardless of how long participants slept was highly satisfactory in $61 \%$ and about $47 \%$ of participants spend 6-7 sleeping hours. Segmented sleeping among the participants has a positive impact on academic performance which was both, directly and indirectly, related to the amount of time spent by the participants in their studies.

Conclusion: Segmented sleep is the most natural way of treating sleep deprivation among the adolescent. Therefore, the segmented sleep pattern can be inducted in the daily schedule and then modifying to suit the individual's lifestyle.

Keywords: Academic performance, Adolescence, Segmented Sleep, and Sleep deprivation.

\section{Introduction}

Sleep is the natural phenomenon that occurs in

\section{Corresponding Author:}

\section{Mr. Balaji Karunakaran}

Tutor, Department of Anatomy, Saveetha Medical College \& Hospital, Saveetha Institute of Medical \& Technical Sciences, Thandalam, Chennai, Tamil Nadu, India 602105

Mobile No.: 9894763124

e-mail:mevsig@gmail.com every individual when the body and mind undergo a temporary state of rest. The individual is physically dormant and consciousness is suspended where the awareness about the environment is reduced. An average healthy individual spends approximately one-third of the life in sleeping and a normal individual need between seven to nine hours of sleep per day to stay healthy and function properly. Sleep is one of the four basic physiological needs namely hunger, thirst, sex, and sleep. The occurrence of sleep in the individual is still a mystery. Sleep has many functions such as development, growth, differentiation, repair, and learning or memory 
consolidation, which occurs throughout the life span. ${ }^{1}$ Adequate sleep plays an important role in the physical and mental wellbeing of the individual especially enhances the cognitive skills, memory retention, levels of concentration and performance of all the activities. Eight hours of continuous sleep in a day of an individual is considered a normal sleeping pattern and it is also known as monophasic sleep. However, there is also an alternative to normal sleeping patterns, called polyphasic or segmented sleep, which is one or many naps with one core sleep. The factors determining the sleep include sleep patterns, number of naps, core sleep length, and the time intervals. Any disturbance in these factors may lead to sleep deprivation, fails to get required sleep. The prevalence of sleep disorders ranges from $22 \%$ to $65 \%$ in the general population and about $28 \%$ of the medical students have insomnia symptoms. ${ }^{2-4}$ An increased risk of sleep deprivation was reported in medical students. ${ }^{5}$ Adequate sleep is positively linked with the general well-being of the individual and their performance in day to day activities. ${ }^{6,7}$ Sleeping pattern of the medical students is an important factor for successful academic performance. Hence this study was done to investigate the effect of segmented sleeping on the academic performance of medical students.

\section{Method and Materials}

This cross-sectional study was done from 2019 to 2020 academic year in the Department of Anatomy, Saveetha Medical College and Hospital, Saveetha Institute of Medical and Technical Sciences, Chennai after getting institutional ethical committee approval (Approval No: SMC/IEC/2020/03/143). The participants were 300 medical students from phase I and II MBBS. A questionnaire was developed and finalized after the elaborated and repeated discussion which was later circulated among the students. The questionnaire was designed with informed consent and questions enquiring about the demographic profile, total sleep time per day, number of naps in a day, sleep latency,and total study hours per day. The data was then analyzed using Microsoft Excel 2010.

\section{Results}

Out of 300 students, 183 (61\%) were females and 117 (39\%) were males. The mean age was 18.9 years with a range of 18 to 21 years. Among the participating students, $55.9 \%$ stay in the hostel provided by the educational institute itself, $35.3 \%$ are day scholars and $8.8 \%$ resided out-campus such as paying guests and rented houses near the campus.

About $47 \%$ of participants spend $6-7$ sleeping hours, $32 \%$ of participants spend less than 5 hours, $12 \%$ spend about $8-9$ hours and a small number of students $(9 \%)$ spend more than 10 hours in sleeping on weekdays, during weekends, all the participants spend 1-2 hours excess in sleeping (Figure 1).More than half of the participants $(56 \%)$ suffer from slight to moderate delay in sleep induction, time taken to fall asleep during night time and $2 \%$ of the participants suffered a severe delay in sleep induction. The overall quality of the sleep regardless of how long participants slept was highly satisfactory in $61 \%$, moderately satisfactory in $37 \%$, and unsatisfactory in $2 \%$ (Figure 2). Similarly, participants had issues in awakening after sleep (27\%) and awakening during sleep (28\%).

The majority of the participants $(56.1 \%)$ spend less than 5 hours of sleep in a night time. The interesting finding was that $85.1 \%$ of the participants have the habit of sleeping after the college hours and $50 \%$ of them were involved in the post-lunch nap. $32.4 \%$ of the participants know about segmented sleeping. Among the hostelers, $86 \%$ of the participants practice segmented sleep, whereas only $53 \%$ of the day scholars practice it. (Figure 3)

Less number of participants $(11.8 \%)$ practice segmented sleeping and among them, $45.1 \%$ of participants positively responded that segmented sleeping helped to spend extra time in studies.

On comparing different correlates with participants segmented sleeping, it was found that segmented sleeping had a better impact on the academic performance which was both, directly and indirectly, related to the amount of time spent by the participants in their studies. (Table 1) It was also found that segmented sleeping was a better determinant of performance than the monophasic sleep where participants had a single continuous one, regardless of the number of hours spend on sleep.

Participants, who practice monophasic sleep, also had excessive daytime sleepiness $52 \%$ which affected their day to day activities including academic performance. Whereas participants who practice segmented sleeping had reported only $8 \%$ excessive daytime sleepiness. 


\section{Discussion}

Segmented sleeping is human's naturally evolved system, well-practiced by ancestors for a long time which was scientifically proved among Romans. ${ }^{8}$ The study done by Barbato $G$ et al 1994 demonstrated the experiment on 14 individuals in which the body reverts to a segmented sleeping on the induction of removal of modern lighting. ${ }^{9}$ The prevalence of sleep disorders is common in females than males, the gender difference is mainly due to men report adverse effects of sleep quality, whereas females more specifically concentrate on a single symptom. ${ }^{10}$ The sleep pattern among medical students on working days and holidays that students slept for more than 8 hours and there was a significant change in the sleep initiation which happened after midnight and wake up the late morning the next day which is normally seen in adolescents. A shift of about $2 \mathrm{hrs}$ from the normal is seen in these subjects. ${ }^{11-13}$

In 2017, Philips reported negative linear correlations between academic performance and sleep patterns. ${ }^{14}$ Medical students who scored " $A$ " in their academic examination had a higher sleep duration when compared with "B" and "C" scoring students. ${ }^{15}$ This significant difference between the students does not explain the students sleeping pattern whether it was monophasic or segmented sleeping. Hence the increase in mean sleep duration with segmented sleeping may also increase academic performance. The modern trend of participating insocial gathering for recreation especially late-night gatherings lead to change in the sleeping patterns among different classes of the society. Further, monophasic sleep used by those who went to sleep later may still have been the segmented sleeping pattern in their second sleep. ${ }^{16}$

Genzel et al. reported a significant correlation between sleep quality during the semester and the pre-clinical board exam performance among medical students. Further, the sleep quality after the board exams for the next year was investigated where the relationship could not be determined. In another study by the same author also concluded that the relationship between sleep quality during the semester and the academic performance in the board exam could not be determined. ${ }^{17}$ The limitations of this study are excluding a few factors such as academic stress, and personal issues which may affect academic performance. Moreover, this study was conducted with participants of only one medical college which need to be elaborate to more sample size. The results of this study are very peculiar. Most participants with lower academic performance found to have monophasic sleep which with short sleep duration may affect sleep quality, sleep efficiency, and sense of sleepiness almost every day. Participants who practice segmented sleep scored better in academic performance were able to get adequate sleep, better sleep quality. Hence with the proper and correct way of segmented sleeping, the academic performance of the students can be improved.

To summarize, the present study evaluated the relationship between academic performance and segmented sleep. The results could show that sleep quality was linked with academic performance. Medical students who suffer from sleep quality issues, which have so far been underestimated health issues should be addressed with segmented sleep pattern with the proper method to improve the academic performance and overall well-being.

\section{Conclusion}

Sleep deprivation is one of the most common health issues among adolescents in modern society which is also a cause of poor academic performance. Segmented sleeping can be an answer to achieve better academic performance when it is practiced properly. The students, teaching faculties, and health professionals need to understand the role of segmented sleeping to improve academic performance. The educational institution should appoint a student psychologist who is also a sleep expert for counseling the students about the importance of quality sleep by segmented sleep for better academic achievement and a healthy lifestyle.

Table 1: Showing the academic performance of participants based on their sleeping pattern

\begin{tabular}{|c|c|c|}
\hline $\begin{array}{c}\text { Marks in } \\
\text { Percentage }\end{array}$ & $\begin{array}{c}\text { Monophasic sleep } \\
\text { (\%) }\end{array}$ & $\begin{array}{c}\text { Segmented Sleep } \\
\text { (\%) }\end{array}$ \\
\hline$>80$ & 2 & 1 \\
\hline $60-80$ & 12 & 19 \\
\hline $40-60$ & 23 & 28 \\
\hline$<40$ & 9 & 6 \\
\hline
\end{tabular}




\section{No of hours spend in sleeping per day}

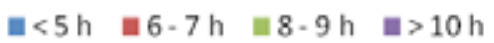

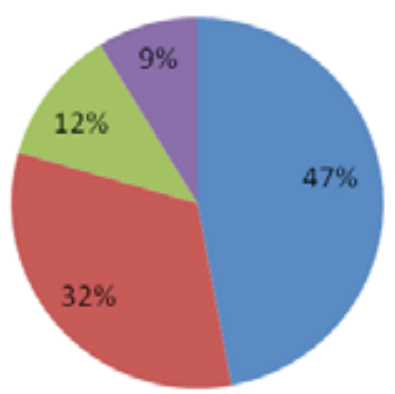

Figure 1: Showing the number of sleeping hours per day among the participants

\section{Quality of sleep}

= highly satisfactory = moderately satisfactory $\quad$ unsatisfactory

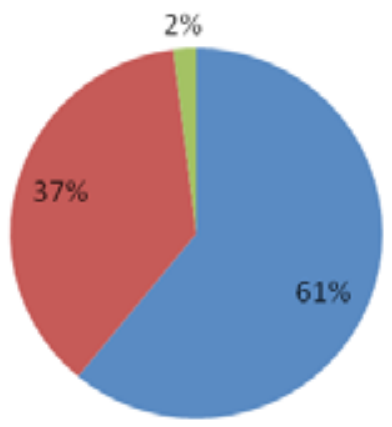

Figure 2: Showing the sleep quality of the participants

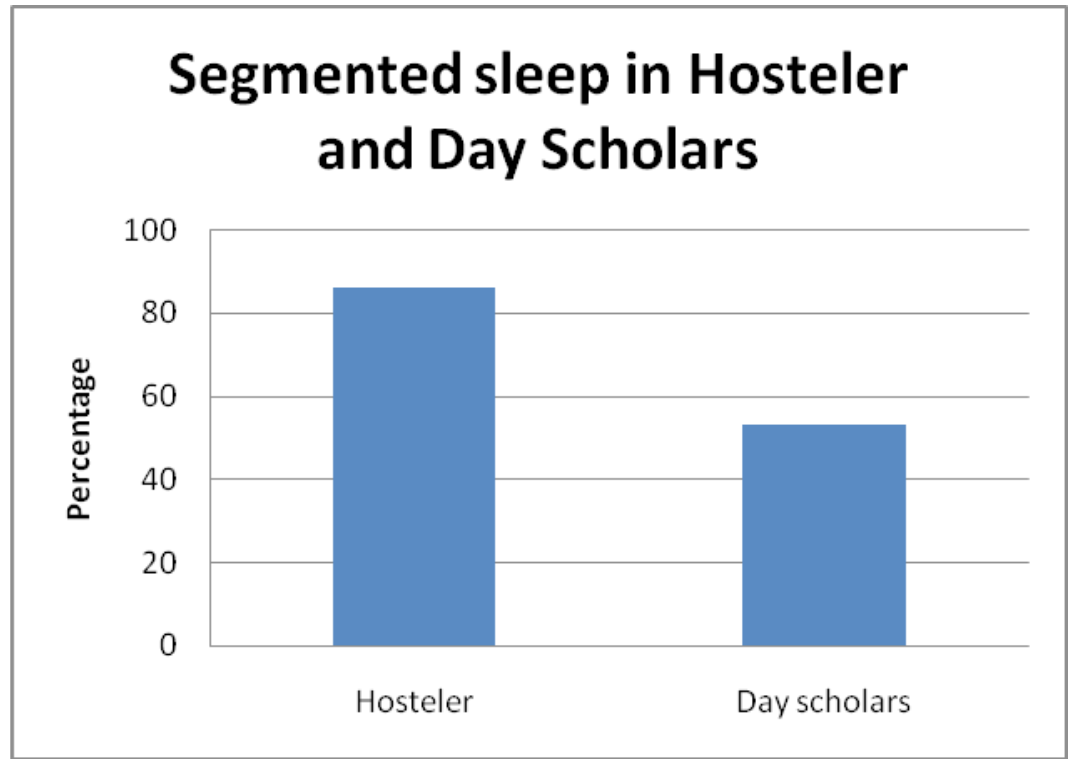

Figure 3: Showing the segmented sleep among hostelers and day scholars 
Acknowledgments: The authors are thankful to all the faculties of the Department of Anatomy, Saveetha Medical College and Hospital, Saveetha Institute of Medical And Technical Sciences (SIMATS), Chennai, India, for the support and gratitude to carry out this work.

Funding: Self-funding

Conflicts of Interest: The authors don't have any conflicting interests.

\section{References}

1. Curcio G, Ferrara M, De Gennaro L. Sleep loss, learning capacity and academic performance. Sleep Med Rev.2006;10(5):323-337.

2. Jewett ME, Dijk DJ, Kronauer RE, Dinges DF. Dose-responserelationship between sleep duration and human psychomotor vigilance and subjective alertness. Sleep. 1999; 22(2):171-179.

3. Roth T, Ancoli-Israel S. Daytime consequences and correlates of insomnia in the United States: Results of the 1991 National Sleep Foundation Survey II. Sleep. 1999; 22:S354-S358.

4. Foley DJ, Monjan AA, Brown SL, Simonsick EM, Wallace RB, Blazer DG. Sleep complaints among elderly persons: An epidemiologic study of three communities. Sleep. 1995; 18(6):425-432.

5. Loayza HMP, Ponte TC, Carvalho CG, Pedrotti MR, Nunes PV, Souza CM,Zanette CB, Voltolini $\mathrm{S}$, Chaves ML. Association between mentalhealth screening by self-report questionnaire and insomnia in medicalstudents. ArqNeuropsiquiatr.2001; 59(2-A):180-185.

6. Parkerson Jr GR, Broadhead WE, Tse CK. The health status and life satisfaction of first-year medical students. Acad Med. 1990; 65(9):586-588.

7. Eliasson AH, Lettieri CJ, Eliasson AH. Early to bed, early to rise! Sleep habits and academic performance in college students. Sleep Breath. 2010; 14(1):71-75.
8. Nissin, Laura. Sleeping culture in Roman literary sources. Arctos. 2015; 49.

9. Barbato G, Barker C, Bender C,Giesen HA,Wehr TA, Extended sleep in humans in 14 hour nights relationship between REM density and spontaneous awakening Electroencephalography and clinical Neurophysiology. 1994; 90: 291-297

10. Niemi PM, Vainiomaki PT. Medical students' distress - quality, continuity and gender differences during a six-year medical programme. Med Teach 2006; 28(2):136-141.

11. Pelayo RP, Thorpy MJ, Glovinsky P. Prevalence of delayed sleep phase syndrome among adolescents. Sleep Res.1998;17:391

12. Regestein QR, Pavlova M. Treatment of delayed sleep phase syndrome. Gen Hosp Psychiatry. 1995; 17:335-345.

13. Ando K, Kripke DF, Ancoli-Israel S. Estimated prevalence of delayed and advanced sleep phase syndromes. Sleep Res. 1995;24:509.

14. Phillips, A. J. K., Clerx, W. M., O’Brien, C. S., Sano, A., Barger, L. K., Picard, R. W., Lockley, S. W., Klerman, E. B., \& Czeisler, C. A. Irregular sleep/ wake patterns are associated with poorer academic performance and delayed circadian and sleep/wake timing. Scientific Reports, 2017; 7(1):3216.

15. Mirghani HO, Mohammed OS, Almurtadha YM, Ahmed MS: Good sleep quality is associated with better academic performance among Sudanese medical students. BMC Res Notes. 2015; 8:706.

16. Ekirch, A. Roger. "Sleep We Have Lost: PreIndustrial Slumber in the British Isles." The American Historical Review 2001; 106(2): 343-86.

17. Alotaibi AD, Alosaimi FM, Alajlan AA, Khalid A. Rahman BA, The relationship between sleep quality, stress, and academic performance among medical students J Family Community Med. 2020; 27(1): 23-28. 